Poster Presentation Abstracts

hospital with 3900 deliveries per year. The aim is to evaluate whether the introduction of a gentamicin prescription, administration and monitoring chart reduces the number of medication errors.

Methods: A retrospective audit looking at all the reported clinical incidents forms involving gentamicin over a 7 year period between 2002 and 2008 .

Results: Since the introduction of the new chart in 2005 there was a $16 \%(0.75)$ reduction in the average annual number of medication errors involving gentamicin from 4.75 to 4.00 . There were no further incidents recorded where the wrong dose of gentamicin was given or where a dose was given despite a high serum concentration. There has also been a $67 \%$ reduction in incidents where a gentamicin level was not monitored as required.

Conclusions: There has been some improvement in the number of gentamicin-based clinical incidents with the introduction of a gentamicin-specific chart, however errors are still occurring. Recommendations include the introduction of regular training on appropriate gentamicin prescribing for new staff and a mandatory yearly update for permanent staff. There is a plan to re-audit this yearly, with consideration of electronic prescribing.
482

\section{SAFETY AND EFFICACY OF VELAGLUCERASE ALFA IN GAUCHER DISEASE TYPE 1 PATIENTS PREVIOUSLY TREATED WITH IMIGLUCERASE}

G. Grabowski ${ }^{1}$, G. Pastores², R. Mardach ${ }^{3}$, C. Eng ${ }^{4}$, L. Smith ${ }^{5}$, P. Fernhoff 6 , J. Charrow ${ }^{7}$, M. Heisel-Kurth ${ }^{8}$, N. Longo ${ }^{9}$, W. Rhead ${ }^{10}$, P. Harmatz ${ }^{11}$, A. Mehta ${ }^{12}$, P. Giraldo ${ }^{13}$, A. Tylki-Szymanska ${ }^{14}$, A. Zimran ${ }^{15}$, E. Crombez ${ }^{16}$

${ }^{1}$ Cincinnati Children's Hospital Medical Center, Cincinnati, OH, ${ }^{2} \mathrm{NYU}$ School of Medicine, New York, NY, ${ }^{3}$ Kaiser Permanente Medical Center, Los Angeles, CA, ${ }^{4}$ Baylor College of Medicine, Houston, TX, ${ }^{5}$ Children's Mercy Hospital, Kansas City, MO, ${ }^{6}$ Emory University, Decatur, GA, ${ }^{7}$ Children's Memorial Hospital, Chicago, IL, ${ }^{8}$ Children's Hospitals of Minnesota, Minneapolis, MN, ${ }^{9}$ University of Utah, Salt Lake City, UT, ${ }^{10}$ Children's Hospital of Wisconsin, Milwaukee, WI, ${ }^{11}$ Children's Hospital Oakland, Oakland, CA, USA, ${ }^{12}$ Royal Free Hospital, London, UK, ${ }^{13}$ Hospital Universitario Miguel Servet, Zaragoza, Spain, ${ }^{14}$ Children's Memorial Health Institute, Warszawa, Poland, ${ }^{15}$ Shaare Zedek Medical Center, Jerusalem, Israel, ${ }^{16}$ Shire Human Genetic Therapies, Cambridge, MA, USA

Objective: To examine safety and efficacy of velaglucerase alfa every other week in patients with Gaucher disease previously receiving imiglucerase.

Methods: This open-label, multicenter, 12-month study, enrolled 41 patients $\geq 2$ years to receive velaglucerase alfa at a dose equalling their prior imiglucerase regimen, with infusions administered over 1-hour.

Results: Forty patients received study drug (18 male, 22 female; 4 previously splenectomized; age range, $9-71$ years, $25 \%<18$ years). Median prior imiglucerase use was 67 months (range 22-192 months). Velaglucerase alfa doses were: $\leq 22.5 \mathrm{U} /$ $\mathrm{kg} \quad(\mathrm{n}=14), 22.5-37.5 \mathrm{U} / \mathrm{kg} \quad(\mathrm{n}=12), 37.5-52.5 \mathrm{U} / \mathrm{kg}$ $(n=7)$, and $>52.5 \mathrm{U} / \mathrm{kg}(\mathrm{n}=7)$. Velaglucerase alfa was generally well tolerated with most AEs of mild or moderate severity. No patient experienced a lifethreatening $A E ; 7$ severe AEs (in 5 patients), and 5 treatment-emergent serious AEs (in 4 patients) were reported. One patient discontinued due to a hypersensitivity reaction; this patient did not have $\lg G$ or $\lg E$ antibodies. No patients developed $\lg G$ antibodies to velaglucerase alfa. Clinical parameters 
were sustained at therapeutic levels through 1 year (table).

\begin{tabular}{|l|c|c|c|c|c|}
\hline & $\mathrm{n}$ & $\begin{array}{c}\text { Base- } \\
\text { line } \\
\text { median }\end{array}$ & $\begin{array}{c}\text { Mean } \\
\text { change } \\
\text { or \% } \\
\text { change } \\
\text { from } \\
\text { baseline } \\
\text { to } \\
\text { month } \\
12\end{array}$ & $90 \%$ Cl & $\begin{array}{c}\text { Clini- } \\
\text { cally } \\
\text { signif- } \\
\text { icant } \\
\text { cutoffs }\end{array}$ \\
\hline $\begin{array}{l}\text { Hemoglobin } \\
\text { (g/dL) }\end{array}$ & 40 & 10.8 & -0.1 & -0.3, & $-1,1$ \\
\hline $\begin{array}{l}\text { Platelet } \\
\text { count } \\
\text { (x10\%) }\end{array}$ & 40 & 162 & $7.0 \%$ & $\begin{array}{c}0.5 \%, \\
13.5 \%\end{array}$ & $\begin{array}{c}-20 \%, \\
20 \%\end{array}$ \\
\hline $\begin{array}{l}\text { Normalized } \\
\text { liver volume } \\
\text { (\% of body } \\
\text { weight) }\end{array}$ & 40 & 1.9 & $0.0 \%$ & $-2.6 \%$, & $-15 \%$, \\
\hline $\begin{array}{l}\text { Normalized } \\
\text { spleen } \\
\text { volume (\% } \\
\text { of body } \\
\text { weight) }\end{array}$ & 36 & 0.5 & $-5.6 \%$ & $\begin{array}{c}-10.8 \%, \\
-0.4 \%\end{array}$ & $15 \%$ \\
\hline
\end{tabular}

[Clinical parameters]

Conclusions: Adult and pediatric Gaucher disease patients, previously treated with imiglucerase for $\geq 6$ months, were successfully transitioned to velaglucerase alfa, with stability in clinical disease measures over 12 months.

Velaglucerase alfa is approved in the U.S.

\section{3}

\section{PROMOTING EARLY CHILDHOOD LITERACY IN PAEDIATRIC CLINICS: REACH OUT AND READ (ROR) IRELAND PILOT STUDY}

L. Brennan ${ }^{1,2}$, A. Nicholson ${ }^{1,2}$, Y. Masuya ${ }^{2}$, A. Carroll ${ }^{1,2}$, E. Molloy ${ }^{2}$, T. Clarke ${ }^{1,2,3}$

${ }^{1}$ Paediatrics, The Children's University Hospital Temple Street, ${ }^{2}$ Royal College of Surgeons in Ireland, ${ }^{3}$ Paediatrics, The Rotunda Hospital, Dublin, Ireland

Background: Regularly reading aloud with young children encourages early literacy. ROR is an inexpensive, internationally well established programme that successfully promotes language development by training healthcare providers to encourage reading aloud and providing them with books to give to young patients (Rotunda Hospital: $€ 2500$ provides over 1000 books / annum).

Aim: Pilot study evaluating first ROR programme in Ireland

Methods: Convenience samples of families with children $<3$ years, followed by neonatologists in Dublin maternity hospital outpatient department, surveyed regarding literacy orientation. Responses compared pre-ROR $(n=38)$ \& post-ROR $(n=38)$, sub-analysed using medical cardholder status as marker for socio-economic disadvantage. Anonymous surveys assessing paediatricians' \& nurses' opinions of programme.

Results: Survey results for medical card holders Pre-ROR \% / Post-ROR \%; 55.6 / 93.3 (Fischer $\mathrm{p}=0.02$ ) had books for their child, $0 / 37$ (Fischer $p<0.01$ ) read at bedtime, $0 / 31.3$ (Chi square $p<$ 0.01 ) listed reading as a favourite activity, 5.6 / 25 (Fischer $p=0.13$ ) reported staff discussed language development. Median number of books and minutes spent reading / day increased from 4.5 to 9 and 10 to 30 respectively (Wilcox $p=0.07 \& p=0.21$ ). Of $14 / 16$ eligible staff who completed survey, all recommend ROR to colleagues; $12 / 14$ felt programme should be continued; 11/14 regularly used ROR. Of those, all enjoy and would continue using ROR. Since ROR, 8/11 discussed language development more and $7 / 11$ felt more knowledgeable on language development.

Conclusion: ROR, an inexpensive and effective literacy promotion programme, was successfully incorporated into an Irish paediatric outpatient department. 